\title{
Studies on "Effect of Organic Nitrogen Source on Production of Antifungal Substance from Clostridium Species"
}

\section{Sharma $P^{*}$ and Thakur RL}

Department of Microbiology, Sardar Bhagwan Singh Post Graduate Institute of Biomedical Science and Research, Dehradun, India

*Corresponding author: Prateek Sharma, MSc, Department of Microbiology, Sardar Bhagwan Singh Post Graduate Institute of Biomedical Science and Research, Dehradun, Uttarakhand, India, Tel: +919773670772; E-mail: shubhsharma009@gmail.com

Received date: January 5, 2018; Accepted date: January 22, 2018; Published date: January 25, 2018

Copyright: (C) 2018 Sharma P, et al. This is an open-access article distributed under the terms of the Creative Commons Attribution License, which permits unrestricted use, distribution, and reproduction in any medium, provided the original author and source are credited.

\begin{abstract}
Objective: In this study, antifungal substances were isolated from different soil bacteria like Clostridium species in the presence of different nitrogen sources. The isolated antifungal substances were then tested against Aspergillus and Trichoderma species to analyse their potential activity.

Methodology and Results: Antifungal substances were isolated from different soil bacterial species and it was observed that only Clostridium species was suitable to isolate active antifungal substances against Aspergillus and Trichoderma species in the presence of different nitrogen sources. Peptone and yeast extract were good nitrogen sources to isolate most active antifungal substances from Clostridium species. Agar well diffusion method was used to measure the zone of inhibitions and the maximum zone of inhibition was found up to $22 \mathrm{~mm}$.
\end{abstract}

Conclusion: Yeast extract and Peptone were found to be good nitrogen sources for the growth of Clostridium sp. YLC and antibiotic production.

Keywords: Antifungal Substance; Antibiotic; Clostridium sp.; Trichoderma

\section{Introduction}

Fungi are eukaryotic organism and therefore the drugs used for treating bacterial infections are in effective against them. The fungi are much more complex organism then bacteria, and are more closely related to humans and animals which are also eukaryotes. Bacteria which are prokaryotic, have a variety of physiologic and structural properties unique to their kingdom and therefore appropriate drug targets available for the action of antifungal are limited. However, despite these similarities fungi have unique characteristics, distinct from mammalian hosts allowing for selective targeting of therapeutic drugs. Although a large number of infections are caused by fungi, due to above mentioned properties, the number of antifungal agents available for treatment of systemic fungi diseases. The classes of currently available antifungal drugs include the polyenes (amphotericin and nystien) which bind to ergosterol in the cell membrane, flucytosine, a pyrimidine analogue, azoles and other inhibiters of ergosterol synthesises and gresiofulvin which interferes with microtubules assembly. During recent years opportunistic fungal infections have increased particularly in aids patients, cancer patients and those who have received organ transplants. Opportunistic fungal infections caused by strains resistant of antifungal agents are frequent in hospitals patients. Several other fungi are capable of making the fungal drugs ineffective by modifying them probably by hydroxylation. Moreover, fungi are slow growers are naturally resistant to antifungal drugs and therefore the risk of serious diseases increases when the patient has debilitating diseases of receiving immune suppressive treatment. The alarming increase in fungal diseases in immunecompromised patients requires effective treatment of these infections.
The cutaneous mycoses are superficial fungal infections of the skin, hair or nails. Essentially no living tissue is invaded, however, a variety of pathological changes occur in the host because of the presence of the infectious agent and/or its metabolic products. The principle aetiological agents are dermatophytic moulds belonging to the genera Microsporum, Trichophyton and Epidermophyton which cause ringworm or tinea of the scalp, glabrous skin and nails; Malassezia fufur, a lipophilic yeast responsible for pityriasis versicolor, follicular pityriasis, seborrhoeic dermatitis and dandruff and Candida albicans and related species, causing candidiasis of skin, mucous membranes and nails. The usual approach to the management of cutaneous infections is to treat with topical agents if possible, but nail and hair infections, widespread dermatophytosis and chronic non-responsive yeast infections are best treated with oral antifungal agents. Topical agents include Nystatin; the Imidazoles such as Clotrimazole, Miconazole, Econazole, Ketoconazole and Bifonazole; Terbinafine and Tolnaftate. Oral agents include Griseofulvin, Ketoconazole, Fluconazole, Itraconazole and Terbinafine. The development of antifungal therapy is problematic because fungal cells are much more similar to human cells. Many drugs that inhibit or kill fungi are also toxic for humans. Although therapy of human's mycosis continues to improve, currently this therapy mainly encompasses amphotericin B and azoles. These agents are not ideal because amphotericin treatment is associated with serve side effects and the azoles possess a fungi-static rather fungicidal action. Opportunistic fungal infection is an emerging problem particularly in immune-compromised patients.

A number of effective antifungal drugs are on hand, but like many other infectious microorganism, pathogenic fungi have find way to develop resistance against the antifungal drugs. The antifungal principle is fungistatic against several diverse species of fungi and shows particularly strong activity filamentous fungi, many of which are serious plant pathogen. Fengycin is an antifungal lipopeptide complex 
Citation: Sharma P, Thakur RL (2018) Studies on "Effect of Organic Nitrogen Source on Production of Antifungal Substance from Clostridium Species". J Antimicrob Agents 4: 1000157. doi:10.4172/2472-1212.1000157

Page 2 of 7

produced by Bacillus subtilus strain F-29-3 [1]. It inhibits filamentous fungi but is ineffective against yeast and bacteria [2] have found a novel phospholipid antibiotic (named Bacilysocin) which accumulates within (or associated with) the cells of Bacillus subtilus 168 and determined the structure by nuclear magnetic resonance and mass spectrometry analyses [3] identify and characterize an antifungal compound produced by Bacillus subtilus YM 10-20 which prevents spore germination of Penicillium roqueforti. An antifungal protein, with a molecular mass of $41.9 \mathrm{kDa}$, and designated as Bacisubin, was isolated from a culture of Bacillus subtilus strain B-916 [4]. Al-Ajlani $\mathrm{MM}$ et al. has investigated commercial medium (Pharm-media) for the production of surfactin by Bacillus subtilus MZ-7 [5]. Candida spp. Specially have emerged as important and opportunistic pathogens in individuals with compromised immunity specially those suffering aids, cancer patients treated with chemotherapy, transplant recipients on immunosuppressive drugs and patients with advanced diabetes [6]. Multi-drug resistance (MDR) is serious complication during treatment of opportunistic fungal infection that frequently afflicts immune compromised individuals [7]. C. glabrata which exhibits strong MDR is an important complication of fungal infection [8] and it is emerging as clinically important fungal pathogen account for about $20-24 \%$ of Candida blood infections [6]. Therefore, search for new antibiotic continues unabated and identification of new antifungal that act on novel molecular targets is especially desirable.

\section{Materials}

\section{Organism of present study}

Clostridium $s p$. YLC was isolated from soil and was found to show inhibitory action against fungal species such as Aspergillus spp., Trichoderma sp.

\section{Source of chemical and microbiological media components}

All the chemicals and media components used were purchased from -MERCH PVT LTD., Bombay, HIMEDIA PVT. LTD., Bombay and LOBA CHEMIE PVT, LTD, Bombay.

\section{Moulds culture}

The fungal cultures were obtained from the culture collection of Department of Microbiology, SBS (PG) Institute of Biomedical Science and Research, Balawala, Dehradun. The fungal cultures were usedAspergillus flavus, Aspergillus nigar, Aspergillus fumigatus.

\section{Media used}

\section{Sabouraud dextrose agar (SDA)}

Peptone -10.0 gm/lit

Dextrose - $40.0 \mathrm{gm} / \mathrm{lit}$

Agar-15.0 gm/lit

$\mathrm{PH} \quad-5.6 \mathrm{gm} / \mathrm{lit}$

\section{Nutrient agar}

Peptone - 5.0 gm/lit

Beef extract - $3.0 \mathrm{gm} / \mathrm{lit}$
Sodium chloride - $5.0 \mathrm{gm} / \mathrm{lit}$

Agar - 15.0 gm/lit

$\mathrm{PH} \quad-7.0 \mathrm{gm} / \mathrm{lit}$

\section{Sucrose broth}

Sucrose - $50.0 \mathrm{gm} / \mathrm{lit}$

Peptone - $10.0 \mathrm{gm} / \mathrm{lit}$

Yeast extract - $5.0 \mathrm{gm} / \mathrm{lit}$

Sodium chloride - $5.0 \mathrm{gm} / \mathrm{lit}$

$\mathrm{MgSo}_{4} .7 \mathrm{H}_{2} \mathrm{O}-0.5 \mathrm{gm} / \mathrm{lit}$

$\mathrm{MnSo}_{4} .7 \mathrm{H}_{2} \mathrm{O}-0.5 \mathrm{gm} / \mathrm{lit}$

$\mathrm{CaCo}_{3}-3.0 \mathrm{gm} / \mathrm{lit}$

Tween $80-0.10 \mathrm{gm} / \mathrm{lit}$

$\mathrm{PH} \quad-6.2 \mathrm{gm} / \mathrm{lit}$

\section{Potato dextrose agar}

Infusion from peeled potatoes - $200.0 \mathrm{gm} / \mathrm{lit}$

Dextrose - $20.0 \mathrm{gm} / \mathrm{lit}$

Agar - 15.0 gm/lit

$\mathrm{PH}-5.6 \mathrm{gm} / \mathrm{lit}$

\section{Bacterial culture isolation}

In this process first we take soil sample from nearby land or field and dry it. Then make serial dilution to isolate desired bacterial culture in 10-1 to $10-5$ conc. And make agar plate for spreading these dilutions.

After 24 hrs incubation period we got different species of bacteria. From those species we identified desired bacterial species that is Clostridium sp.

\section{Maintenance of cultures}

Clostridium sp. YLC was maintained on nutrient agar medium slants while moulds cultures were maintained on Potato dextrose agar medium and Sabouraud dextrose agar medium. All cultures were stored at $4^{\circ} \mathrm{C}$ in refrigerator. All organisms were sub cultured after every two months.

\section{Methods}

For bacterial culture isolation, we are using serial dilution method.

Before begin the experiment of assessing antifungal activity, the agar medium was sterilized at $121^{\circ} \mathrm{C}$ for 15 minutes and sterilized $10 \%$ tartaric acid solution, at the rate of $1 \mathrm{ml} / 100 \mathrm{ml}$ of the medium was added to lower the $\mathrm{pH}$ to 3.5 whenever required.

\section{Assessing Antifungal Activity by Agar well diffusion method}

Preparation of cell free supernatant: Freshly grown culture of the isolated was inoculated in Sucrose medium and was incubated at $37^{\circ} \mathrm{C}$ under anaerobic condition. After $72 \mathrm{hrs}$ of incubation $3 \mathrm{ml}$ of the broth was transferred to sterilized centrifuge tubes and centrifuged at 7000 
rpm for 10 minutes. The supernatant was transferred to a sterilized tube. This supernatant was used to assay the antifungal activity against Aspergillus $s p$. The biomass pallet was used to measure the growth of isolate.

Preparation of agar plates and well in the agar: $25 \mathrm{ml}$ of molten Sabouraud dextrose agar cooled at $50^{\circ} \mathrm{C}$ was poured in to the sterilized Petri plates and allowed to solidify. Four and five wells of $6 \mathrm{~mm}$ diameter and placed at equal distance were made with plates with the help of a sterilized borer. A mould cell suspension was spread over the agar surface of the plates prepared above. Plates were placed at $37^{\circ} \mathrm{C}$ for 15 minutes in order to make the agar surface dry. Then $0.1 \mathrm{ml}$ of supernatant added in to the wells with help of sterilized pipette. The plates were kept in upright position in an incubator at $27^{\circ} \mathrm{C}$ until filtrate diffused into the agar (at least $12 \mathrm{hrs}$.). Further the plates were incubated in inverted position for 72 to $96 \mathrm{hrs}$. The plates were observed for the zone of inhibition around the wells (if any) and diameters of zones were measured in $\mathrm{mm}$.

Measurement of growth of the Clostridium sp. YLC: Growth of the culture was measured in term of O.D. at $620 \mathrm{~nm}$. The biomass pellet settled at the bottom of the centrifuged tube after transfer of supernatant was dissolved in distilled water with the help of vortex shaker and centrifuged again. The supernatant was thrown and pellet was re-suspended in $3 \mathrm{ml}$ of distilled water. The O.D of this uniform suspension was measured at $620 \mathrm{~nm}$ using digital spectrophotometer 169 (systonic).

Effect of nitrogen source: Nitrogen source that is yeast extract, beaf exract, peptone, and malt extract and tryptone were supplemented in basal medium at concentration of $0.5 \%, 1.0 \%, 1.5 \%, 2.0 \%, 2.5 \%$ and studied for their effect on the growth of Clostridium sp. YLC and antibiotic production by it.

\section{Results}

Clostridium sp. YLC a soil isolate was found to show inhibitory activity against same pathogenic and non-pathogenic fungi which include Microsporum canis, Trichophyton subsum, Aspergillus niger and Trichoderma $s p$. The medium showed very good production of the antifungal substance when it was grown in a medium that contained glucose as a carbon source and yeast extract $(0.25 \%)$ and Trypton $(0.5 \%)$ as nitrogen source. In the present study the effect of some selected nitrogen source viz yeast extract, beef extract, peptone, malt extract and tryptone. The effect of individual nitrogen source was studied by incorporation different concentration of the source in the basal medium.

\section{Effect of different concentration of yeast extract}

Yeast extract was incorporated in the basal medium (Glucose $0.1 \%$ and $0.1 \%$ skimmed milk powder) in the concentration range at 0.5 to $2.5 \%$. The results have been compiled in the (Table 1). The data in the table shows that yeast extract when present in the medium as role nitrogen source proved to be a good nitrogen source for the growth and synthesis of antifungal substance only at higher concentration of 2.0 and $2.5 \%$. At lower concentration of 0.5 and $1.0 \%$ less growth of the producer organism and less antifungal substance production occurred. The growth O.D obtained at these concentrations was 0.608 and 0.845 respectively as compared to 0.936 in case of control. Likewise yield of antifungal substance in term of zone of inhibition were 16 and $19 \mathrm{~mm}$ respectively in comparison to $20 \mathrm{~mm}$ in control. It was at yeast extract concentration of $1.5 \%$ that a zone diameter of $20 \mathrm{~mm}$ was obtained which was equal to those observed in control. However, when the yeast extract concentration was increased to $2.0 \%$ a zone diameter of $22 \mathrm{~mm}$ was recorded which was greater than that observed in case of control. Similar zone diameter was also obtained when the yeast extract concentration in the medium was increased to $2.5 \%$ (Figure 1).

\begin{tabular}{|l|l|l|}
\hline $\begin{array}{l}\text { Conc. of yeast extract } \\
(\%)\end{array}$ & $\begin{array}{l}\text { Growth of organism (O.D. } \\
\text { at } \mathbf{6 2 0} \mathbf{~} \mathbf{m})\end{array}$ & $\begin{array}{l}\text { Zone of inhibition } \\
(\mathbf{m m})\end{array}$ \\
\hline Control & 0.936 & 20 \\
\hline 0.5 & 0.603 & 16 \\
\hline 1 & 0.845 & 19 \\
\hline 1.5 & 0.88 & 20 \\
\hline 2 & 1.117 & 22 \\
\hline 2.5 & 1.048 & 22 \\
\hline
\end{tabular}

Table 1: Effect of different concentrations of yeast extract on production antifungal substance by Clostridium sp. YLC.

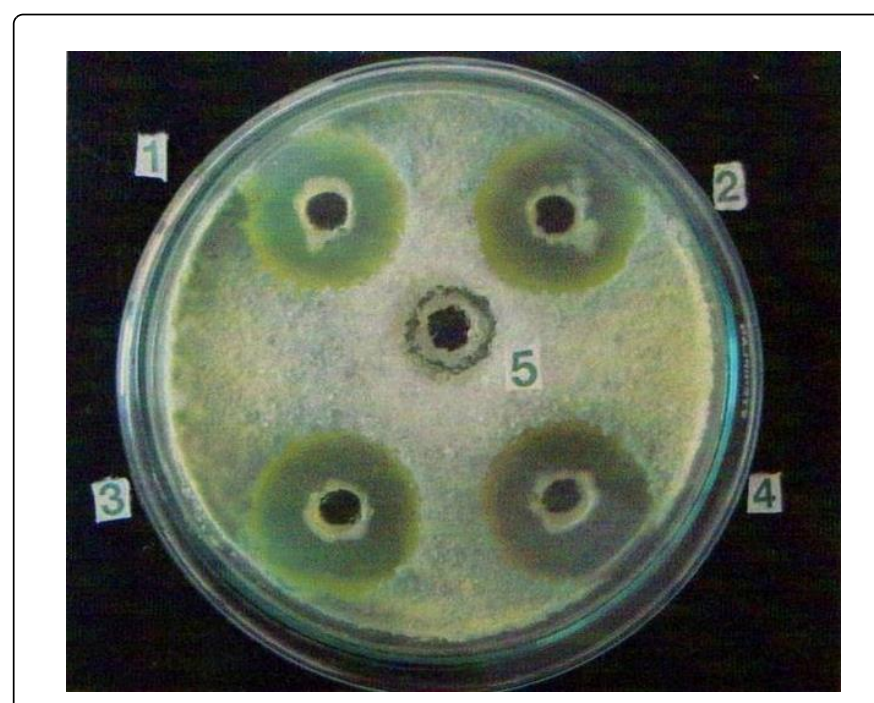

Figure 1: Zone of inhibition obtained when Yeast extract was used as nitrogen source in different concentration of 1.) $2.5 \%, 2$.) $1.5 \%$, 3.) $2.0 \%$, 4.) $1.0 \%$ and 5.) $0.5 \%$.

\section{Effect of different concentration of beef extract}

Table 2 shows the trends of growth of Clostridium sp. YLC and synthesis of antifungal substance when beef extract in the concentration range of 0.5 to $2.5 \%$ was incorporated in the basal medium. It can be inferred from the Figure 2 that beef extract served as a poor sour for the growth of Clostridium $s p$. YLC and production of this nitrogen source tested, growth O.D recorded were less than that obtained in control. This growth O.D range from 0.524 to 0.958 in comparison to 0.988 recorded in control. At lower beef extract concentration of 0.5 and $1.0 \%$ growth was very less with value of 0.524 and 0.634 in comparison to 0.988 in case of control medium. However, at higher concentration of 1.5 and $2.0 \%$ the growth O.D were closer to that of control. Thus growth O.D of 0.905 and 0.952 were obtained at these concentrations than 0.988 in case of control. Similar trend was 
Citation: Sharma P, Thakur RL (2018) Studies on "Effect of Organic Nitrogen Source on Production of Antifungal Substance from Clostridium Species". J Antimicrob Agents 4: 1000157. doi:10.4172/2472-1212.1000157

Page 4 of 7

also observed in synthesis of antifungal substance. At all concentration the antifungal activity recorded was less than that obtained in case of control. Maximum production of antifungal substance was formed to occur at beef extract concentration of $2.0 \%$ where a zone diameter of $15 \mathrm{~mm}$ was recorded in comparison to $20 \mathrm{~mm}$ in control.

\begin{tabular}{|l|l|l|}
\hline $\begin{array}{l}\text { Conc. of beef extract } \\
(\%)\end{array}$ & $\begin{array}{l}\text { Growth of organism } \\
\text { (O.D. at } \mathbf{6 2 0} \mathbf{~ n m})\end{array}$ & $\begin{array}{l}\text { Zone of inhibition } \\
(\mathbf{m m})\end{array}$ \\
\hline Control & 0.988 & 20 \\
\hline 0.5 & 0.524 & 14 \\
\hline 1 & 0.634 & 16 \\
\hline 1.5 & 0.905 & 18 \\
\hline 2 & 0.952 & 19 \\
\hline 2.5 & 0.841 & 17 \\
\hline
\end{tabular}

Table 2: Effect of different concentrations of beef extract on production antifungal substance by Clostridium sp. YLC.

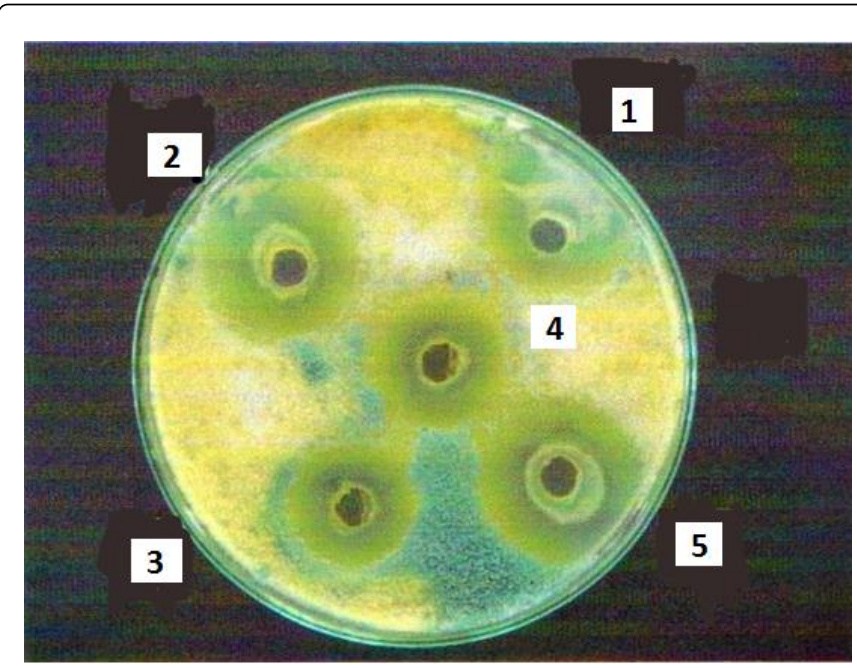

Figure 2: Zone of inhibition obtained when beef extract was used as nitrogen source in different concentrations of: 1.) $2.5 \%, 2$.) $2.0 \%, 3$.) $0.5 \%, 4$.) $1.0 \%$ and 5 .) $1.5 \%$.

\section{Effect of different concentration of peptone}

Table 3 shows the data obtained for the growth of Clostridium $s p$. YLC and synthesis $\mathrm{f}$ antifungal substance when peptone in the concentration range of when peptone in the concentration range of 0.5 to $2.5 \%$ was used as a nitrogen source. It can be inferred from the table that peptone like yeast extract served in good nitrogen source both for growth of the producer organism as well for synthesis of antifungal substance. However, better growth and antibiotic yield were obtained when the concentration of peptone in the medium was above $1.5 \%$. Lower concentration of 0.5 and $1.0 \%$ were not sufficient enough to support antibiotic production equivalent to the obtained in case of control medium. At these two concentration the zone of inhibition obtained against Trichoderma spp. were 16 and $20 \mathrm{~mm}$. respectively which were either less than or equal to that obtained in control. However, at peptone concentration of $1.5,2.0$ and $2.5 \%$ the zone diameter obtained were 21, 22 and $22 \mathrm{~mm}$ respectively. Thus antibiotic production was better than that observed in case of control (Figure 3).

\begin{tabular}{|l|l|l|}
\hline $\begin{array}{l}\text { Conc. of peptone } \\
(\%)\end{array}$ & $\begin{array}{l}\text { Growth of organism (O.D. } \\
\text { at } 620 \mathbf{n m})\end{array}$ & Zone of inhibition $(\mathbf{m m})$ \\
\hline Control & 0.964 & 20 \\
\hline 0.5 & 0.544 & 16 \\
\hline 1 & 0.789 & 20 \\
\hline 1.5 & 0.855 & 21 \\
\hline 2 & 0.97 & 22 \\
\hline 2.5 & 1.056 & 22 \\
\hline
\end{tabular}

Table 3: Effect of different concentrations of peptone on production antifungal substance by Clostridium $s p$. YLC.

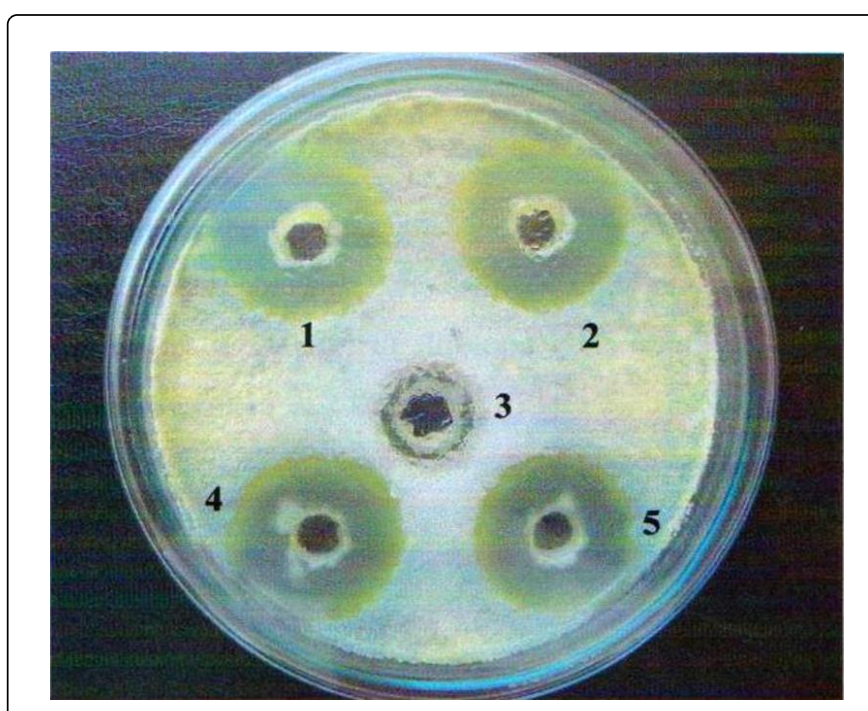

Figure 3: Zone of inhibition obtained when peptone was used as nitrogen source in different concentration.1). 2.5\%,2). 2.0\%, 3). $0.5 \%, 4) .1 .5 \%$ and 5$) .1 .0 \%$.

\section{Effect of different concentration of malt extract}

The data obtained for the growth of Clostridium $s p$. YLC in terms of O.D at $620 \mathrm{~nm}$ and antibiotic production in term of zones of inhibition have been depicted in Table 4. This table shows that malt extract when used as a role nitrogen source in a concentration range of 0.5 to $2.5 \%$ did not serve as a good nitrogen source both for the growth of the test organism and for antibiotic production. At all concentration of malt extract tested growth recorded was less than that obtained in control. At lower concentration of very less growth of the test organism occurred as indicated by the low growth O.D of 0.371 and 0.400 in comparison to 0.886 observed on control. The growth increased with increasing concentration of malt extract. This increase continue up to malt extract concentration of $2.0 \%$ where a maximum O.D of 0.663 was recorded but it was still sufficiently less than that observed in case of control medium (0.886). There was slight decrease in growth at malt extract concentration of $2.5 \%$ as the O.D decreased to 0.646 . Similar trend was also observed in synthesis of antifungal substance. Sufficient 
less antifungal activity than control were recorded at all the concentration of malt extract in corporate in the medium. The zone diameter ranged from $13 \mathrm{~mm}$ to $18 \mathrm{~mm}$ in comparison to $21 \mathrm{~mm}$ obtained in control medium. Maximum zone diameter of $18 \mathrm{~mm}$ was recorded when malt extract concentration in the medium was 2.0-2.5\%.

\begin{tabular}{|l|l|l|}
\hline $\begin{array}{l}\text { Conc. of malt extract } \\
(\%)\end{array}$ & $\begin{array}{l}\text { Growth of organism } \\
\text { (O.D. at } \mathbf{6 2 0} \mathbf{~} \mathbf{m})\end{array}$ & Zone of inhibition $(\mathbf{m m})$ \\
\hline Control & 0.886 & 21 \\
\hline 0.5 & 0.375 & 13 \\
\hline 1 & 0.4 & 15 \\
\hline 1.5 & 0.591 & 16 \\
\hline 2 & 0.663 & 18 \\
\hline 2.5 & 0.646 & 18 \\
\hline
\end{tabular}

Table 4: Effect of different concentrations of Malt extract on production antifungal substance by Clostridium sp. YLC.

\section{Effect of different concentration of tryptone}

The influence of tryptone on the growth of the organism and the synthesis of antifungal substance was more or less similar to that observed on case of malt extract. Very less growth of the organism was observed at lower tryptone concentration of 0.5 and $1.0 \%$ with growth O.D of 0.392 and 0.438 respectively in comparison to an O.D of 0.871 obtained in case of control medium in Figure 2. The growth of the organism increased concentration of tryptone. Thus at tryptone concentration of $2.5 \%$ the O.D increased to 0.751 which was the maximum O.D obtained of all the tryptone concentration tested. However, it was slightly less than that recorded in the control medium (0.876). Almost similar trend was observed in synthesis of antifungal antibiotic. At lower concentration of 0.5 and $1.0 \%$ the zone diameter obtained were $13 \mathrm{~mm}$ and $14 \mathrm{~mm}$ respectively in comparison to 20 $\mathrm{mm}$ observed in control. Among the different concentration of tryptone tested maximum zone at $20 \mathrm{~mm}$ was obtained at $2.5 \%$ level which was similar to that recorded in case of control (Table 5).

\begin{tabular}{|l|l|l|}
\hline $\begin{array}{l}\text { Conc. of tryptone } \\
(\%)\end{array}$ & $\begin{array}{l}\text { Growth of organism (O.D. } \\
\text { at } 620 \mathbf{n m})\end{array}$ & Zone of inhibition $(\mathbf{m m})$ \\
\hline Control & 0.871 & 20 \\
\hline 0.5 & 0.392 & 13 \\
\hline 1 & 0.438 & 14 \\
\hline 1.5 & 0.546 & 16 \\
\hline 2 & 0.668 & 18 \\
\hline 2.5 & 0.751 & 20 \\
\hline
\end{tabular}

Table 5: Effect of different concentrations of Tryptone on production antifungal substance by Clostridium sp. YLC.

\section{Summary and Conclusion}

Clostridium sp. was isolated from soil. The isolate was found to show inhibition a Trichoderma $s p$. Further, studies showed that it was capable of inhibiting some potential fungal pathogens such as Microsporum canis, Trichophyton ruburm and opportunistic pathogen Aspergillus niger. In the present study effect of some selected nitrogen sources viz yeast extract, beef extract, peptone, malt extract and tryptone on production of antifungal substance has been investigated.

Yeast extract when incorporated as a role nitrogen source in the basal medium, was found to be a good nitrogen source both for growth of Clostridium sp. YLC and for production of antifungal substance. Particularly it was more effective at higher concentration of 2.0 to $2.5 \%$ where both growth of the organism and antibiotic yield better than that of control were obtained.

Beef extract when incorporated in the medium at concentration of 0.5 to $2.5 \%$ proved to be a poor source for the production of antifungal substance, although good growth of the organism was observed particularly at higher concentrations. Thus a maximum zone of inhibition of $19 \mathrm{~mm}$ was obtained when the level of beef extract in the medium was $2.0 \%$. However, this antibiotic yield was less than that recorded in case of control medium (zone diameter $20 \mathrm{~mm}$ ).

Peptone like yeast extract was a good source for the growth of Clostridium sp. YLC as well as for antibiotic production particularly at higher concentration of 1.5 to $2.5 \%$. A yield better than that in control was recorded at $1.5 \%$ peptone level and a zone diameter of $21 \mathrm{~mm}$ was obtained in comparison to $20 \mathrm{~mm}$ in control. Highest antibiotic production occurred at peptone concentration of $2.0 \%$ where a zone diameter of $22 \mathrm{~mm}$ was recorded.

Malt extract was found to be a poor source both for the growth of the producer organism as well as for antibiotic production. Growth O.Ds. Were appreciable less than that obtained in case of control medium. Thus growth O.D. varied from 0.371 to 0.663 in comparison to 0.886 in control. Likewise, antibiotic yields were also and poor zone diameters of 13 to $18 \mathrm{~mm}$ were obtained as compared to $21 \mathrm{~mm}$ recorded in control.

Tryptone like malt extract was found to be poor source both for growth of organism and antibiotic production. Biomass and antibiotic yields were slightly better than those recorded in case of malt extract. Growth optical densities varied from 0.392 to 0.725 and zone diameter from 13 to $20 \mathrm{~mm}$. Thus tryptone concentration of $2.5 \%$ produced a zone diameter which was similar to that obtained in case of control.

Thus yeast extract and peptone were found to be good nitrogen sources for the growth of Clostridium sp. YLC and antibiotic production. Beef extract stimulated good growth but antibiotic yields were comparatively poor. Malt extract and tryptone were found to be poor sources both for growth of the organism and antibiotic production.

\section{Discussion}

Nitrogen sources are the major nutrients that are essential for the growth of the living organisms. Nitrogen is required for the synthesis of amino acids which are the building blocks of the proteins. Proteins are the major constituents of the microbial biomass. Enzymes are also proteins. Moreover, nitrogen is also required for the synthesis of nucleotides and cofactors. Thus the nature and concentration of nitrogen sources present in the medium affect the growth of an organism which in term influences the synthesis of metabolites such as antibodies and other metabolites. Thus for maximum synthesis of a particular metabolites the presence of an appropriate nitrogen sources 
and its optimum level is essential. Generally, organic nitrogen sources such as peptones, tryptone, etc. which are rich in amino acids and peptides are better sources than the inorganic nitrogen sources. Therefore, in the present study effect of different nitrogen sources viz yeast extract, beef extract, peptone, malt extract and tryptone was studied on the growth of Clostridium sp. YLC and antibiotic production by it.

\section{Influence of yeast extract}

Yeast extract when incorporated in the basal medium in a concentration range of 0.5 to $2.5 \%$ was found to stimulate good antibiotic production particularly at higher concentration in the concentration range of 1.5 to $2.5 \%$. In this concentration range zone diameters of 20 to $22 \mathrm{~mm}$ were obtained which were either similar to or bigger than that observed in control medium.

The production of antifungal substance by Lactobacillus acidophilus was studied by Batish et al. [9]. A yeast extract concentration of $1.0 \%$ was found to be optimum for maximum production of a substance. In another study on production of an antifungal substance by Bacillus $s p$. AT-1, [10] has reported that a concentration of $1.0 \%$ was optimum for the synthesis of antibiotic. However, in the present study a higher concentration of $2.0 \%$ was found optimum.

\section{Effect of beef extract}

Beef extract was found to support good growth of Clostridium $s p$. YLC. However, it was not a good nitrogen source for the synthesis of antifungal substance. Maximum synthesis of the antibiotic was found to occur when the beef extract concentration in the medium was $2.0 \%$. A zone diameter of $19 \mathrm{~mm}$ was produced at this concentration in comparison to $20 \mathrm{~mm}$ in control.

Effect of different concentration of beef extract was studied on production of antifungal substance by Bacillus sp. SS-1 [11]. Beef extract was found to be a poor source. Similarity assessed the influence of different nitrogen sources on synthesis of antifungal antibiotic by Bacillus sp. AT-1 and reported that in comparison to tryptone and yeast extract, beef extract was found a poor nitrogen source [12].

\section{Influence of peptone}

Peptone like yeast extract, was found to be a good nitrogen source both for growth of Clostridium $s p$. YLC and antibiotic production. Growth of the organism was comparable to that in control or slightly better, when the peptone concentration in the medium was in the range 1.5 to $2.5 \%$. Maximum antibiotic production was also recorded in the same range of concentration where zone diameters of 21 to 22 $\mathrm{mm}$ were record in comparison to $20 \mathrm{~mm}$ in control.

Effect of different nitrogen sources on antibiotic production by Bacillus sp. AT-1 was studied [10]. Peptone was found to be a poor nitrogen source for antibiotic production. However, while studying the effect of different nitrogen sources on production an antifungal antibiotic by Bacillus sp. SS-1, have reported that peptone was the best source for synthesis of antibiotic and a concentration of $1.5 \%$ in the medium was found optimum for this purpose [11].

\section{Influence of malt extract}

Malt extract was found to be a poor source both for growth of Clostridium sp. YLC and antibiotic synthesis by it. Growth O.Ds. Of
0.371 to 0.665 were obtained in the concentration range of 0.5 to $2.5 \%$ of malt extract in comparison to 0.886 in control. Likewise, zone diameters of $13-18 \mathrm{~mm}$ were recorded in comparison to $21 \mathrm{~mm}$ in control.

Effect of different concentrations of malt extract was studied on production of an antimicrobial substance by Bacillus sp. PR-1. Malt extract was found to be a good source for antibiotic production and a concentration of $1.5 \%$ was found to be optimum [13].

\section{Effect of tryptone}

Use of tryptone in the medium as role nitrogen source although resulted in poor biomass yields in comparison to control medium but antibiotic production was as good as in control. However, it occurred at higher concentration of $2.5 \%$. A zone diameter of $20 \mathrm{~mm}$ was obtained at this concentration which was similar to that observation in case of control medium.

Batish et al. studied the effect of different nitrogen sources on production of an antifungal substance by Lactobacillus lactis subspecies Diacetylactis. Tryptone was found to be a good source in comparison to peptone and yeast extract [14]. Have found tryptone to be the best nitrogen source for synthesis of antifungal substance by Bacillus sp. AT-1 [12].

\section{References}

1. Vanittanakom N, Loeffler W, Koch U, Jung G (1986) Fengycin is an antifungal lipopeptide complex produced by Bacillus subtilus strain F29-3. J Antibiot (Tokyo) 39: 888-901.

2. Tamehiro N, Hosoya YO, Okamoto S, Ubukata M, Hamada M, et al. (2002) Bacilysocin, a novel phospholipid antibiotic produced by Bacillus subtilis 168. Antimicrob Agents Chemother 46: 315-320.

3. Chitarra GS, Breeuwer P, Nout MJ, Aelst AC, Rombouts FM, et al. (2003) Antifungal compound produced by Bacillus subtilus YM 10-20. J Appl Microbiol 94: 159-166.

4. Liu Y, Chen Z, Ng TB, Zhang J, Zhou M, et al. (2007) Bacisubin, an antifungal protein with ribonuclease and hemagglutinating activities from Bacillus subtilis strain B-916. Peptides 28: 553-559.

5. Al-Ajlani MM, Sheikh MA, Ahmad Z, Hasnain S (2007) Production of surfactin from Bacillus subtilis MZ-7 grown on pharmamedia commercial medium Microb Cell Fact 6: 17.

6. Pfaller MA, Diekema DJ, Colombo AL, Kibbler C, Richardson M (2006) Candida rugosa, an emerging fungal pathogen with resistance to azoles: geographic and temporal trends from the ARTEMIS DISK antifungal surveillance program. J Clin Microbiol 44: 3578-3582.

7. Magiorakos AP, Srinivasan A, Carey RB, Carmeli Y, Falagas ME, et al. Multidrug-resistant, extensively drug-resistant and pandrug-resistant bacteria: An international expert proposal for interim standard definitions for acquired resistance. Clin Microbiol Infect 18: 268-281.

8. Klesper DR (2006) Clostridium glabrata exhibits strong MDR is an important complication of fungal infection. Ther Adv Hematol 2: 231-247.

9. Roy U, Batish VK, Grover S, Neelakantan S (1996) Production of antifungal substance by Lactococcus lactis subsp. lactis CHD-28.3. International J Food Microbiol Prod 32: 27-34.

10. Yun-Feng YE, Qi-Qin LI, Gang FU, Anand G (2008) identification of antifungal substance (iturin a2) produced by Bacillus subtilis B47 and its effect on southern corn leaf blight. J Integrat Agri 11: 90-99.

11. Saxena S (2006) Effect of different concentration of beef extract on production of antifungal substance by Bacillus sp. SS-1. J Antimicro 15: 18-25. 
Citation: Sharma P, Thakur RL (2018) Studies on "Effect of Organic Nitrogen Source on Production of Antifungal Substance from Clostridium Species". J Antimicrob Agents 4: 1000157. doi:10.4172/2472-1212.1000157

Page 7 of 7

12. Anand J (2008) Assessing the antifungal potentials of Bacillus sp. AT-1 and effect of nitrogen sources on production of the substance. H.N.B Garhwal University, Srinagar, Garhwal.

13. Gupta R (2005) Evolution of organic nitrogen sources for antibiotic production by Bacillus sp. PR-1. H.N.B Garhwal University, Srinagar, Garhwal.
14. Batish VK, Lal R, Grover S (1990) Studies on environmental and nutritional factors, on production of antifungal substance by Lactobacillus acidophilus. R Food Microbial 7: 199-206. 